\title{
Effects of the IGF-1/PTEN/Akt/FoxO signaling pathway on male reproduction in rats subjected to water immersion and restraint stress
}

\author{
PAN HUANG $^{1 *}$, ZHENGRONG ZHOU ${ }^{1 *}$, FANGXIONG SHI $^{2}$, GENBAO SHAO $^{1}$, \\ RAN WANG $^{1}$, JINTIAN WANG ${ }^{1}$, KANGXIN WANG ${ }^{1}$ and WEI DING ${ }^{3}$ \\ ${ }^{1}$ Department of Pathology, School of Medicine, Jiangsu University, Zhenjiang, Jiangsu 212013; \\ ${ }^{2}$ Laboratory of Animal Reproduction, College of Animal Science and Technology, Nanjing Agricultural University, \\ Nanjing, Jiangsu 210095; ${ }^{3}$ Department of Animal Husbandry and Veterinary Medicine, \\ Jiangsu Polytechnic College of Agriculture and Forestry, \\ Jurong, Jiangsu 212400, P.R. China
}

Received October 8, 2015; Accepted October 3, 2016

DOI: $10.3892 / \mathrm{mmr} .2016 .5880$

\begin{abstract}
The aim of the present study was to determine the effects of the insulin-like growth factor 1 (IGF-1)/phosphatase and tensin homologue deleted on chromosome 10 (PTEN)/Akt/forkhead box (FoxO) signaling pathway on male reproduction in rats subjected to water immersion and restraint stress (WRS). Sperm morphology, sperm malformation rate, and serum testosterone concentration were analyzed following WRS. In addition, the expression levels and immunolocalization of IGF-1, PTEN, Akt and FoxO proteins, as well as the rate of cell apoptosis in rat testes, were investigated. The results indicated that sperm malformation rate, serum testosterone concentration, and the number of terminal deoxynucleotidyl transferase dUTP nick end labeling-positive cells were increased in the testes after WRS. Furthermore, IGF-1 and FoxO1 proteins were predominantly localized in the sperm cytoplasm during the late stages of spermatogenesis. FoxO1 protein was also localized in Leydig cell cytoplasm. PTEN and total Akt proteins were predominantly expressed in the cytoplasm of Leydig cells and spermatogonia. PTEN protein was also detected in vascular endothelial cells. In addition, $I G F-1$, PTEN, Akt1, Akt2, FoxO3 and FoxO4 gene expression levels were upregulated following WRS, and peaked after $7 \mathrm{~h}$ of WRS. During the recovery period, the expression
\end{abstract}

Correspondence to: Dr Wei Ding, Department of Animal Husbandry and Veterinary Medicine, Jiangsu Polytechnic College of Agriculture and Forestry, 3 Jushu Road, Jurong, Jiangsu 212400, P.R. China

E-mail: qishi6598@126.com

${ }^{*}$ Contributed equally

Key words: IGF-1, FoxO, signaling pathway, testis, stress, rat levels of these genes gradually returned to normal levels. The present study demonstrated that WRS induced sperm damage in the testes. In addition, the results indicated that the IGF-1/PTEN/Akt/FoxO signaling pathway may serve an anti-stress role in the testes of rats subjected to WRS.

\section{Introduction}

It is well known that stress causes considerable harm to human health and also impairs livestock production. The hypothalamic-pituitary-adrenal (HPA) axis and the sympathetic nervous system (SNS) are stimulated by exogenous and endogenous factors, which may induce stress reactions, thus triggering compensatory physiological responses (1). After the HPA axis and SNS receive stimulatory signals, the secretion of glucocorticoids and catecholamines is increased. Glucocorticoids exhibit feedback inhibition on HPA function through binding with receptors in the pituitary gland, hypothalamus and prefrontal cortex (2). Long-term stress may cause depression and neurological disorders in animals and humans. Behavioral stress is a major risk factor for several clinical disorders, including cardiovascular disease, metabolic disease and psychosis. Furthermore, stress is associated with mental disorders, such as anxiety and personality disorders. Several studies have demonstrated that stress can increase the incidence of diseases, including gastroenteritis (3-5). However, research on stress and male reproduction is fairly limited.

The insulin-like growth factor 1 (IGF-1)/phosphatase and tensin homologue deleted on chromosome 10 (PTEN)/Akt/forkhead box (FoxO) signal pathway serves important roles in various cells and tissues $(6,7)$. IGF-1 has a role in signal transduction by binding to its receptor, and subsequently regulating cellular processes via phosphorylation (8). Phosphoinositide 3-kinase (PI3K)/Akt is the key downstream signaling pathway of IGF-1, which regulates cell proliferation, growth and apoptosis. The Akt family, also known as protein kinase B, has three members: Akt1, Akt2 
and Akt3, which have high homology and specific expression patterns. Akt1 and Akt2 are widely expressed in tissues and cells; however, Akt3 is only expressed in specific tissues and cells (9). PTEN is a tumor suppressor gene with phosphatase activity, which regulates cell proliferation cycles and inhibits cell migration. PTEN is the phosphatase of phosphatidylinositol $(3,4,5)$-trisphosphate (PIP3), reverses the transformation of phosphatidylinositol 4,5-bisphosphate to PIP3, inhibits PI3K phosphorylation, suppresses the activity of Akt and downstream kinases, arrests the cell cycle at $\mathrm{G}_{1}$ phase, and negatively regulates cell growth (10). FoxO is one of the most important target genes of the downstream IGF-I/PTEN/Akt signaling pathway, and includes FoxO1, FoxO3a, FoxO4 and FoxO6 (11). FoxO is regulated by Akt phosphorylation (12), following which its nuclear export is inactivated; therefore, the activation of apoptotic genes downstream of FoxO is inhibited. During this process, the nuclear-cytoplasmic trafficking of FoxO is ultimately involved in the regulation of cell apoptosis (13).

Water immersion and restraint stress (WRS) has been reported to increase the expression of IGF-1 in rat gastric mucosa tissue, and regulate cell apoptosis of the gastric mucosa via the PI3K/Akt signal pathway (14). IGF-1 is a specific anti-apoptotic factor present in mouse Leydig cells during embryonic developmental stages (15). IGF-1 also serves an important role in the proliferation and differentiation of mouse Sertoli cells, through the PI3K/Akt signal pathway during the adolescent stage $(16,17)$. In the proliferation and differentiation of Sertoli cells of 20-day-old mice, follicle-stimulating hormone (FSH) can activate the PI3K/Akt signaling pathway. The role of PTEN in spermatogenesis also remains controversial. Wu et al hypothesized that PTEN may have a role in the late stage of sperm development (18). Dupont et al demonstrated that estrogen induced testicular tumors in mice via the PTEN/Akt signal pathway (19). Similarly, Kimura et al revealed that PTEN gene knockout could lead to testicular tumors in mice (20). The expression levels of PTEN were gestational age-specific in the fetal rat testes, thus suggesting that PTEN may have a key role in fetal rat testis development (21). However, Huang et al reported that PTEN did not serve a role in regulating spermatogenesis in mice (22). Previous reports have also focused on the role of FoxO in male reproduction. FoxO1 has a crucial role in the initiation of spermatogenesis; however, two other members, FoxO3a and FoxO4, do not appear to serve any roles (23-26). Therefore, the IGF-1/PTEN/Akt/FoxO signaling pathway may have an important role in male reproduction.

In the present study, immunohistochemistry (IHC), terminal deoxynucleotidyl transferase dUTP nick end labeling (TUNEL) staining and quantitative polymerase chain reaction (qPCR) were conducted to investigate the effects of WRS on male reproductive function in rats. In addition, the possible roles of the IGF-1/PTEN/Akt/FoxO signaling pathway were investigated following WRS.

\section{Materials and methods}

Reagents. Antibodies against FoxO1 (cat. no. 9462), FoxO3a (cat. no. 9467), FoxO4 (cat. no. 9472) and total Akt (cat. no. 9272 were purchased from Cell Signaling Technology,
Inc. (Beverly, MA, USA). Antibodies against IGF-1 (cat. no. BA0939) were purchased from Wuhan Boster Biological Technology Co., Ltd. (Wuhan, China). Antibodies against PTEN (cat. no. sc-9145) were obtained from Santa Cruz Biotechnology, Inc. (Dallas, TX, USA). Avidin-biotin complex kits were obtained from BioGenex (Fremont, CA, USA) and 3,3'-diaminobenzidine tetrachloride (DAB) was purchased from Sigma-Aldrich (Merck Millipore, Darmstadt, Germany). SYBR Premix Ex Taq (cat. no. DRR420A) and PrimeScript ${ }^{\mathrm{TM}}$ RT reagent kit with gDNA Eraser (cat. no. DRR047S) were purchased from Takara Biotechnology Co., Ltd. (Dalian, China). One-step TUNEL Apoptosis Assay kit (cat. no. C1088) and Caspase 3 Activity Assay kit (cat. no. C1116) were obtained from Beyotime Institute of Biotechnology (Haimen, China). All other chemicals were purchased commercially and were reagent grade.

Animals and sample collection. A total of 50 intact male Sprague-Dawley rats (age, 9-11 weeks; weight, 200-220 g) were obtained from Qinglongshan Experimental Animal Breeding Farm (Nanjing, China) for use in the present study. Uniform commercial diets used in the present study were also purchased from Qinglongshan Experimental Animal Breeding Farm. Rats received regular rat chow and tap water ad libitum, and were housed individually at $25^{\circ} \mathrm{C}$ and $65-70 \%$ humidity under a 12-h:12-h light/dark cycle. WRS models were established as described in our previous study (27). For each time point, six rats were sacrificed after 0,3 and $7 \mathrm{~h}$ of WRS, whereas others were fed normally $1 \mathrm{~h}$ after WRS and six were sacrificed at several time points (4, 8 and 15 days) after the end of $7 \mathrm{~h}$ WRS. Rats were anesthetized with ether inhalation and sacrificed by cervical dislocation. In order to examine protein localization and conduct TUNEL staining, testicular samples were immediately removed from anesthetized rats and fixed in $4 \%(\mathrm{v} / \mathrm{v})$ paraformaldehyde at room temperature overnight. In addition, testicular samples were stored in liquid nitrogen for the analysis of gene expression and caspase- 3 activity. All procedures were designed in accordance with generally accepted ethical standards for animal experimentation and the guidelines established by the institutional animal care and use committee of Jiangsu University (Jiangsu, China).

Epididymal sperm reserves. Epididymal sperm morphology was observed as described previously (28). Briefly, cauda epididymides were sampled, and gently minced in $2.0 \mathrm{ml}$ phosphate-buffered saline (PBS). The minced material was then homogenized for $1 \mathrm{~min}$, filtered through a nylon mesh screen, and the filtrate was brought up to a final volume of $10 \mathrm{ml}$ with PBS. Epididymal sperm morphology was observed under a high power light microscope following Giemsa staining (Shanghai Gefan Biotechnology, Co., Ltd., Shanghai, China) at room temperature. The morphology of 200 sperm per rat was evaluated. The sperm malformation rate percentage was then calculated using a random selection of one optical area, in which 200 sperm were consecutively evaluated for malformation.

Assessment of apoptotic cell number. The testicular samples were embedded in paraffin wax and cut into $7-\mu \mathrm{m}$ sections. The TUNEL staining was performed using a one-step TUNEL 
Table I. Primers used for quantitative PCR analysis.

Gene and sequence

reference

(GenBank no.)

Primer sequence

Size of PCR

product (bp)
Annealing temperature $\left({ }^{\circ} \mathrm{C}\right)$
HPRT (X62085)

IGF-1 (BC086374)

PTEN (NM031606)

Aktl (NM033230)

Akt2 (NM017093)

Akt3 (NM031575)

FoxO1 (NM001191846)

$$
\begin{aligned}
& \text { F:5'-AGTGATGATGAACCAGGTTA-3' } \\
& \text { R:5'-ATTATAGTCAAGGGCATATC-3' } \\
& \text { F:5'-TGGTGGACGCTCTTCAGTTC-3' } \\
& \text { R:5'-GCTTCAGCGGAGCACAGTAC-3 } \\
& \text { F:5'-AGCGTGCGGATAATGACAAG-3' } \\
& \text { R:5'-GGATTTGATGGCTCCTCTACTG-3' } \\
& \text { F:5'-TAGGCATCCCTTCCTTACAG-3' } \\
& \text { R:5'-GCCCGAAGTCCGTTATCT-3' } \\
& \text { F:5'-GAGCCGAGTCCTACAGAATACC-3' } \\
& \text { R:5'-GGCCATCTTTGTCCAGCATA-3' } \\
& \text { F:5'-AACGACCAAAGCCAAATACA-3' } \\
& \text { R:5'-CCCCATTAACATATTCCATCAC-3' } \\
& \text { F:5'-CGTCCTCGAACCAGCTCAA-3' } \\
& \text { R:5'-TTGGCGGTGCAAATGAATAG-3' }
\end{aligned}
$$

556
168
151
269
263
498
292

556

58.0

168

58.0

151

56.0

269

58.0

263

58.0

498

58.0

292

57.4

PCR, polymerase chain reaction; HPRT, hypoxanthine phosphoribosyltransferase 1; IGF-1, insulin-like growth factor 1; PTEN, phosphatase and tensin homolog deleted on chromosome 10; FoxO1, forkhead box protein 10.

Apoptosis Assay kit, as described previously (29). Briefly, testicular cells were counterstained with DAPI (Beyotime Institute of Biotechnology) to label all nuclear DNA, and fragmented DNA was end-labeled with fluorescein isothiocyanate-labeled dUTP using terminal transferase. The sections were then examined under a confocal immunofluorescence microscope (LSM5 PASCAL; Carl Zeiss, Oberkochen, Germany). Sections exposed to DNase I, which causes DNA fragmentation, exhibited intense nuclear staining and were used as positive controls. For negative controls, dUTP was omitted, resulting in uniformly negative staining. Ten optical areas, containing 500-1,000 cells, were counted in each slide under high-power (400x) microscopy and the number of positive cells per area was counted.

Determination of caspase -3 activity. Testicular samples ( $\mathrm{n}=6$ for each treatment) were rinsed with cold PBS, and homogenized on ice in lysis buffer $(3-10 \mathrm{mg} / 100 \mu \mathrm{l})$. Homogenates were transferred to $1.5 \mathrm{ml}$ centrifuge tubes, lysed on ice for $5 \mathrm{~min}$ and centrifuged at $18,000 \mathrm{x} g$ for $10 \mathrm{~min}$ at $4^{\circ} \mathrm{C}$. Caspase- 3 activity was determined in the lysates of testicular samples from rats that underwent various treatments using a caspase-3 activity kit. Briefly, this colorimetric assay is based on hydrolysis of the substrate peptide, Ac-DEVD-pNA, by caspase-3. The released moiety ( $\mathrm{p}$-nitroaniline) has a high absorbance at $405 \mathrm{~nm}$. Therefore, the concentration of p-nitroaniline $(\mu \mathrm{M})$ released from the substrate is calculated from the absorbance values at $405 \mathrm{~nm}$, or from a calibration curve prepared using defined p-nitroaniline solutions (30).

IHC for IGF-1 (1:1,00), PTEN (1:200), total Akt (1:200) and FoxO1 (1:300) was performed on formalin-fixed, paraffin-embedded testicular tissue sections using a standard protocol, as described previously, using DAB and hematoxylin counterstaining $(27,31)$.
RNA extraction, reverse transcription and $q P C R$. Total RNA was extracted from testicular samples using TRIzol ${ }^{\circledR}$ reagent (Invitrogen; Thermo Fisher Scientific, Inc., Waltham, MA, USA). RNA concentration and purity were determined using a spectrophotometer (NanoVue; GE Healthcare, Piscataway, NJ, USA), and the integrity was examined using $1.2 \%$ agarose gels containing $0.1 \%$ ethidium bromide. Total RNA $(1 \mu \mathrm{g})$ obtained from each extraction was reverse transcribed in a $20 \mu 1$ reaction volume using an RT reagent kit with gDNA Eraser according to the manufacturer's protocol. The primers (Thermo Fisher Scientific, Inc.) were designed based on the corresponding gene sequences (Table I). qPCR was performed with a $20 \mu 1$ reaction volume containing $2 \mu 1$ template cDNA, $0.4 \mu \mathrm{l}$ forward/reverse primers, $10 \mu \mathrm{l} 2 \mathrm{X}$ SYBR qPCR mix and $0.4 \mu 1$ ROX reference dye (Takara Bio, Inc., Otsu, Japan). on an ABI 7300 instrument (Applied Biosystems; Thermo Fisher Scientific, Inc.). The PCR conditions were as follows: $95^{\circ} \mathrm{C}$ for $10 \mathrm{~min}$, followed by 45 cycles of denaturation at $95^{\circ} \mathrm{C}$ for $15 \mathrm{sec}$ and $60^{\circ} \mathrm{C}$ for $45 \mathrm{sec}$. Experiments for the detection of all genes, including the housekeeping gene hypoxanthine phosphoribosyltransferase 1 (HPRT), were performed in triplicate. The relative expression levels of the genes tested were calculated using the $2^{-\Delta \Delta \mathrm{Cq}}$ method (32).

Testosterone concentration. Rat blood samples were obtained from the heart and transferred to centrifuge tubes. After $2 \mathrm{~h}$ standing at room temperature, samples were centrifuged at $1,400 \times \mathrm{g}$ for $10 \mathrm{~min}$ at $4^{\circ} \mathrm{C}$ to obtain serum. Testosterone concentrations in the serum were detected using a commercial radioimmunoassay kit (cat. no. DF00008; Nanjing Jiancheng Bioengineering Institute, Nanjing, China) according to the manufacturer's protocols. The cross-reaction rates of this antiserum with progesterone, cortisol, estradiol and dehydroepiandrosterone (Beijing North Institute of Biological 


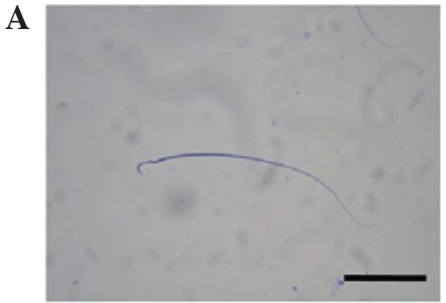

D

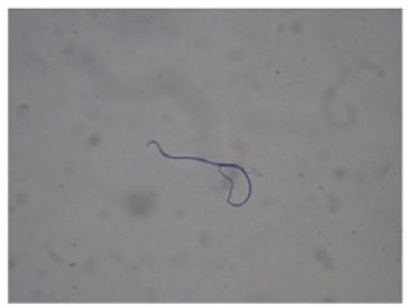

B

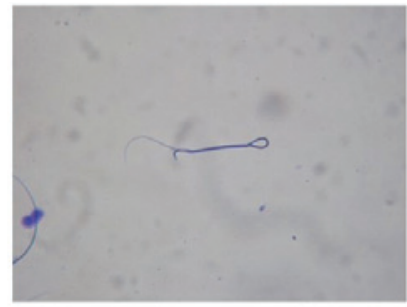

$\mathbf{E}$

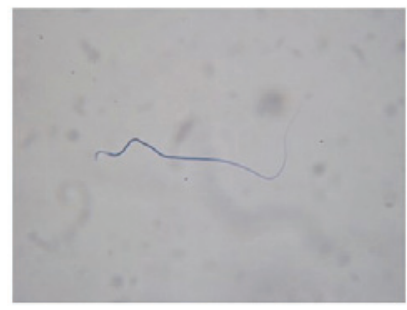

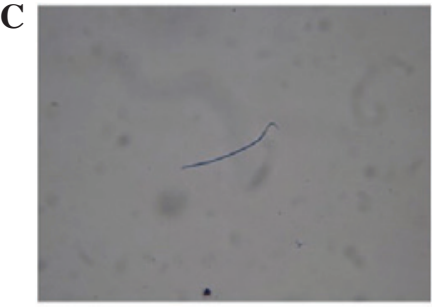

F

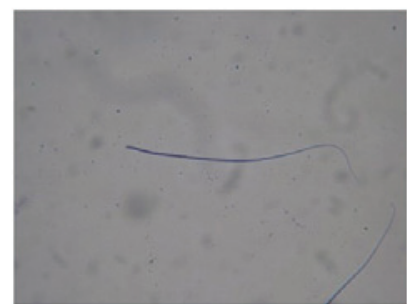

Figure 1. Photomicrographs of sperm from rats subjected to water immersion and restraint stress. (A) Normal morphology; (B-D) tail malformation; (E) malformation in the middle; (F) decapitated sperm. Scale bar=100 $\mu \mathrm{m}$.

Technology, Beijing, China) were $<0.01 \%$. The intra-coefficients of variation for androgen determination in this laboratory were $<9 \%$. Treatments were performed in triplicate, and each experiment was repeated at least three times.

Statistical analysis. SPSS 17.0 software (SPSS, Inc., Chicago, IL, USA) was used to conduct statistical analyses. All experiments were repeated in triplicate, and representative data is presented. Data are presented as the mean \pm standard error of the mean. Data were analyzed using one-way analysis of variance and Fisher's protected least significant difference test. $\mathrm{P}<0.05$ was considered to indicate a statistically significant difference.

\section{Results}

Effects of WRS on sperm malformation rate. Following WRS, sperm in rat epididymis exhibited several morphologies, as follows: Normal morphology (control group, Fig. 1A), malformation in the tail ( $7 \mathrm{~h}$ group, Fig. 1B; $3 \mathrm{~h}$ group, Fig. 1C; 15 day group, Fig. 1D), malformation in the middle $(3 \mathrm{~h}$ group, Fig. 1E), and decapitated sperm (7 h group, Fig. 1F). In addition, WRS increased the sperm malformation rate in the rat epididymis (Fig. 2). When WRS was extended to $7 \mathrm{~h}$, the sperm malformation rate was significantly higher compared with in the other groups $(\mathrm{P}<0.05 ; \mathrm{n}=6)$.

Effects of WRS on serum testosterone concentrations. Testosterone concentrations in the sera of rats 3 and $7 \mathrm{~h}$ after WRS were increased in a time-dependent manner, as compared with the control group $(\mathrm{P}<0.05 ; \mathrm{n}=6)$. On days 4 , 8 and 15 after $7 \mathrm{~h}$ WRS, testosterone concentrations were gradually decreased compared with in the $7 \mathrm{~h}$ WRS group (Fig. 3).

Effects of WRS on apoptosis in rat testes. Testis tissue sections were stained using the TUNEL method, in order to determine the quantity and distribution of apoptotic cells, and examine nuclear condensation and fragmentation (Fig. 4). Sections exposed to DNase I, which causes DNA fragmentation,

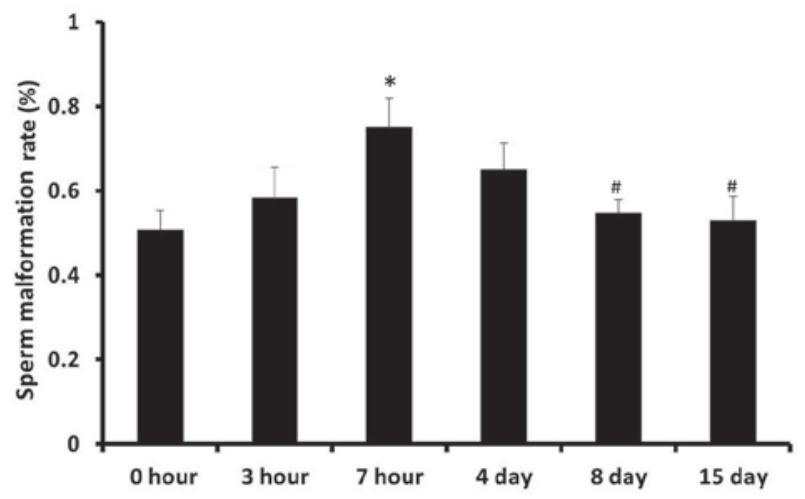

Figure 2. Effects of water immersion and restraint stress on sperm malformation rate. $\mathrm{n}=6$ in each treatment group. ${ }^{*} \mathrm{P}<0.05$ vs. the $0 \mathrm{~h}$ group; ${ }^{\#} \mathrm{P}<0.05$ vs. the $7 \mathrm{~h}$ group.

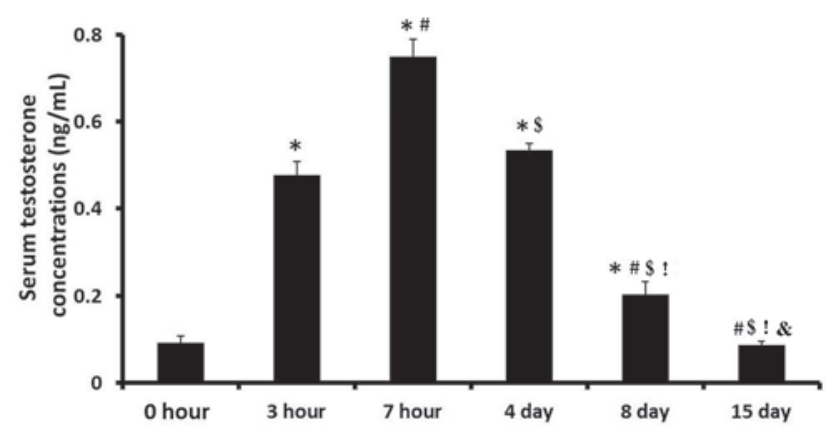

Figure 3. Effects of water immersion and restraint stress on serum testosterone concentrations. $\mathrm{n}=6$ in each treatment group. ${ }^{*} \mathrm{P}<0.05$ vs. the $0 \mathrm{~h}$ group; ${ }^{*} \mathrm{P}<0.05$ vs. the $3 \mathrm{~h}$ group; ${ }^{\$} \mathrm{P}<0.05$ vs. the $7 \mathrm{~h}$ group; ${ }^{\mathrm{P}}<0.05$ vs. the 4 day group; ${ }^{\text {}} \mathrm{P}<0.05$ vs. the 8 day group.

exhibited intense staining of all nuclei and were used as positive controls (data not shown). Sections stained using the described procedure, but without the terminal deoxynucleotidyl transferase enzyme exhibited no staining and were used as negative controls (Fig. 4S-U). In the testis of non-WRS rats, a small amount of labeling was detected, which was predominantly 


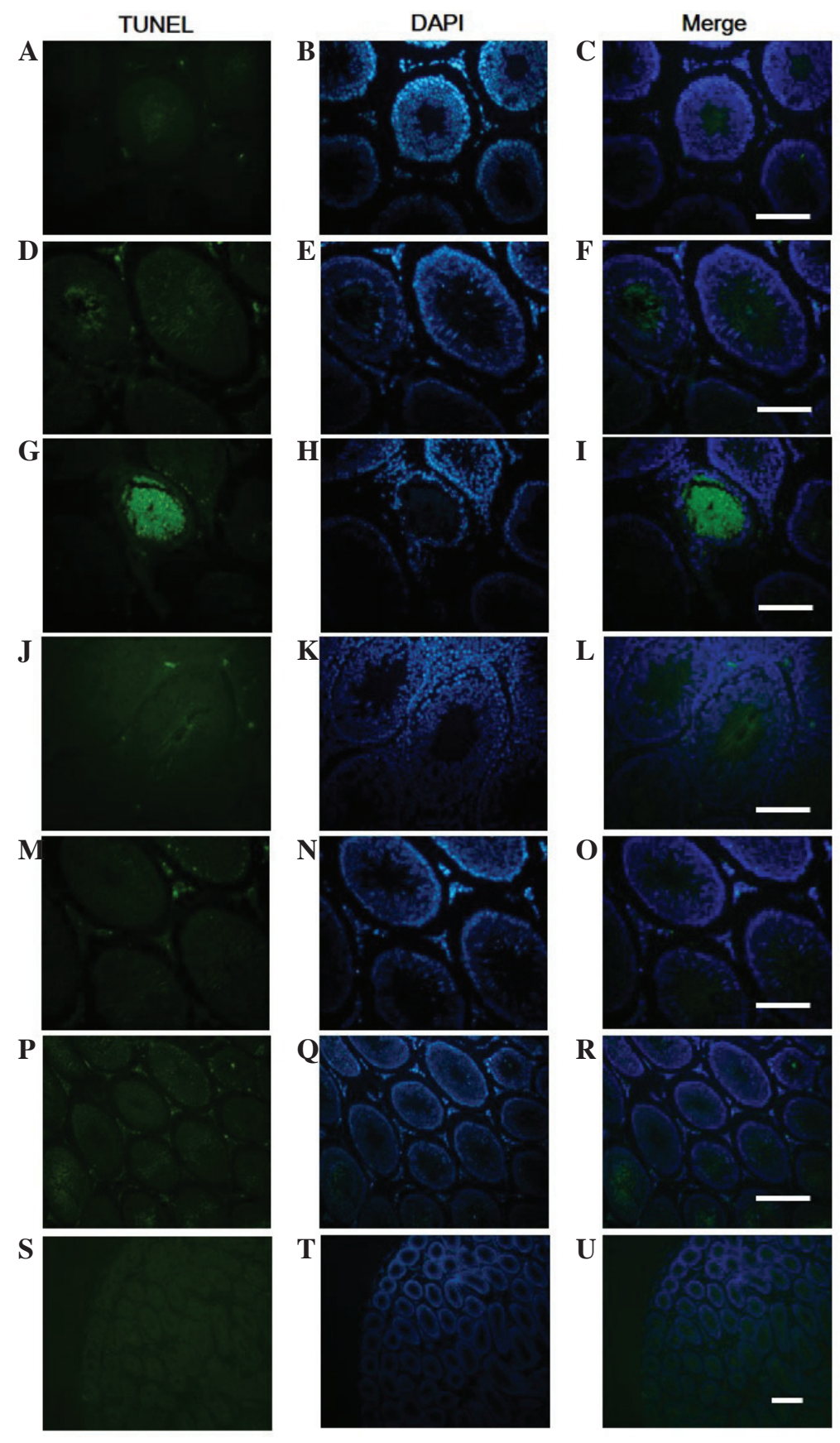

Figure 4. Effects of WRS on apoptotic cells in rat testes. After TUNEL staining, rat testis sections were counterstained with DAPI to label all nuclei. Blue staining indicates DAPI-stained nuclei; green staining indicates TUNEL-positive cells. Images were captured from (A-C) non-WRS control rats; rats following (D-F) $3 \mathrm{~h}$ and (G-I) $7 \mathrm{~h}$ of WRS; rats (J-L) 4 days, (M-O) 8 days and (P-R) 15 days after $7 \mathrm{~h}$ WRS; and (S-U) negative control rats. Scale bar=100 $\mu \mathrm{m}$. WRS, water immersion and restraint stress; TUNEL, terminal deoxynucleotidyl transferase dUTP nick end labeling; DAPI, 4',6-diamidino-2-phenylindole.

concentrated in the cells of late generation spermatogonia in the seminiferous tubules. Scattered TUNEL-positive cells were also detected in Leydig cells (Fig. 4A-C). Compared with in the control group, the distribution of TUNEL-positive cells in rat testis following WRS was not markedly altered (Fig. 4D-R). The number of TUNEL-positive cells in the rat testes subjected to $7 \mathrm{~h}$ WRS was significantly increased, compared with in the control group (Fig. 5A; $\mathrm{P}<0.05 ; \mathrm{n}=6$ ). Subsequently, from day 4 to 15 after $7 \mathrm{~h}$ WRS, the number of TUNEL-positive cells in the testes decreased gradually, and on day 15 , the number of TUNEL-positive cells recovered to normal levels (Fig. 5A).
To confirm cell apoptosis in the testes of WRS rats, caspase- 3 activity was detected in the testes using a colorimetric assay. As shown in Fig. 5B, caspase-3 activity in the testes following WRS was not significantly different compared with in the control group $(\mathrm{P}>0.05)$.

Immunohistochemical localization of IGF-1, PTEN, total Akt and FoxOl in the testes of rats after WRS. To assess the localization of IGF-1, PTEN, total Akt, and FoxO1 in rat testes, sections from WRS rat testes were stained with specific antibodies targeting these proteins (Fig. 6). IGF-1 (3 h group, Fig. 6A; 4 day group, Fig. 6B) and FoxO1 (control group, 

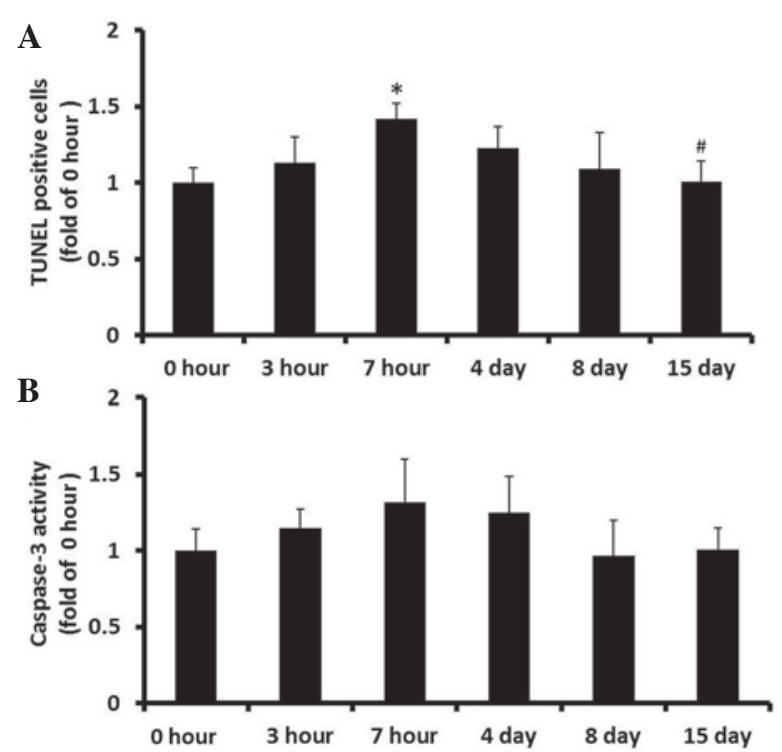

Figure 5. Effects of water immersion and restraint stress on (A) the number of TUNEL-positive cells in rat testes and (B) caspase-3 activity in rat testes. Data (fold of control) are presented as the mean \pm standard error of the mean. $\mathrm{n}=6$ in each treatment group. ${ }^{*} \mathrm{P}<0.05$ vs. the $0 \mathrm{~h}$ group; ${ }^{*} \mathrm{P}<0.05$ vs. the $7 \mathrm{~h}$ group. TUNEL, terminal deoxynucleotidyl transferase dUTP nick end labeling.

Fig. 6E; 7 h group, Fig. 6F) were widely observed in the sperm cytoplasm during late stage spermatogenesis; FoxO1 was also expressed in Leydig cell cytoplasm. In addition, PTEN (15 day group, Fig. 6C) and total Akt (15 day group, Fig. 6D) were localized in Leydig cells and cytoplasm of spermatogonia. PTEN was also detected in vascular endothelial cells.

Relative expression levels of IGF-1, PTEN, Akt1, Akt2, Akt3 and FoxOl in rat testes after WRS. The expression levels of selected genes were analyzed using qPCR. Amplification products were identified through melting curve profile analysis and were confirmed with gel electrophoresis and sequencing. The relative transcript of each target gene was normalized to HPRT (Fig. 7). All selected genes were transcriptionally active. The mRNA expression levels of IGF-1, PTEN, FoxOI and $A k t 2$ were increased in the testes of rats subjected to WRS; the levels reached their peak after $7 \mathrm{~h}$ of WRS. In the recovery phase, the expression levels of these genes gradually dropped to normal levels. In addition, the results indicated that WRS did not affect Aktl and Akt3 gene expression in rat testes.

\section{Discussion}

The WRS rat has been widely used as a model of gastroduodenal mucosal lesions; however, to the best of our knowledge, the effects of WRS on male reproductive function have not been reported. In the present study, the WRS rat was used to investigate the effects of WRS on the reproductive function of adult male rats. The results indicated that WRS increased sperm malformation rate and serum testosterone concentrations, thus suggesting that WRS induced sperm damage in rats. There may be two reasons by which WRS causes damage: i) When generating the WRS model, the water temperature was $20 \pm 2^{\circ} \mathrm{C}$, whereas the room temperature was $30^{\circ} \mathrm{C}$, indicating that the sudden temperature change may cause sperm damage in rats; ii) environmental stress affects the HPA axis function, which may increase testosterone concentration in rats and ultimately affect sperm morphology; however, the specific mechanisms require further study.

The caspase family serves an important role in mediating cell apoptosis, and caspase- 3 serves as a key execution molecule. Caspase-3 normally exists in the form of zymogen (32 kD) in the cytoplasm, which is activated in the early stages of apoptosis. The activated caspase- 3 consists of one large subunit (17 kD) and two small subunits (12 kD), which recognize related substrates in the cytoplasm and nucleus, eventually leading to apoptosis. Creagh et al demonstrated that numerous external stressors induce apoptosis, then inhibit caspase activity (33). In the present study, the effects of WRS on apoptosis in the rat testes were investigated by caspase-3 activity assay and the TUNEL method. The results indicated that WRS increased the number of TUNEL-positive cells, and the TUNEL-positive cells were predominantly distributed in spermatoblasts that were in the late stages of spermatogenesis in the seminiferous tubules; however, caspase-3 activity was not changed. These results suggested that WRS may induce damage to the spermatoblast in the late stages of spermatogenesis.

The IGF-1/PTEN/Akt/FoxO signaling pathway serves critical roles in regulating cell differentiation, migration and apoptosis in various cells and tissues (34). The present study demonstrated that WRS induced rat sperm damage. To confirm whether the IGF-1/PTEN/Akt/FoxO signaling pathway was involved in the anti-damage mechanism of sperm, the localization and expression levels of IGF-1, PTEN, total Akt and FoxO proteins were detected. The results indicated that the IGF-1 protein was widely expressed in sperm cytoplasm, during late stage spermatogenesis. In addition, WRS increased the gene expression levels of $I G F-1$ in rat testes. Similarly, a previous study reported that WRS could increase the expression levels of IGF-1 in rat gastric mucosa, which was able to regulate the downstream PI3K/Akt signaling pathway via cyclooxygenase-2, and participated in regulation of gastric mucosal cell apoptosis (35). Colon et al demonstrated that IGF-1 is a cell-specific anti-apoptotic factor in mouse testes during embryonic development (36). In the newborn period, IGF-1 served an important role in sustentacular cell differentiation and proliferation via the PI3K/Akt signaling pathway in mouse testes $(16,17)$. Unlike previous studies, mature rats were used in the present study, thus suggesting that IGF-1 may serve different roles at different developmental stages of testicular tissue; this requires further investigation. In addition, the gene expression levels of $I G F-1$, which has been recognized as an anti-apoptotic factor, were elevated after WRS, thus suggesting that IGF-1 may be involved in anti-injury mechanisms in sperm.

Several studies have reported that Akt widely participates in the growth and development of testicular tissue and serves a critical role in cell differentiation and proliferation $(16,17,36)$. However, the role of PTEN in spermatogenesis remains controversial. Wu et al demonstrated that PTEN may have a role in the late period of sperm development (18). Dupont et al (19) treated mice with exogenous FSH and estrogen, and reported that FSH controls proliferation and differentiation of Sertoli 
A

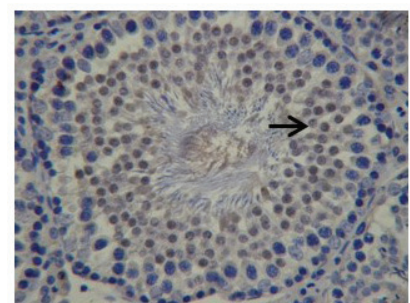

C

$\mathbf{E}$

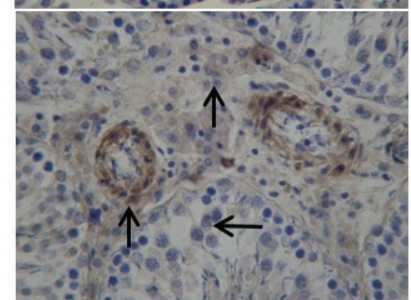

G
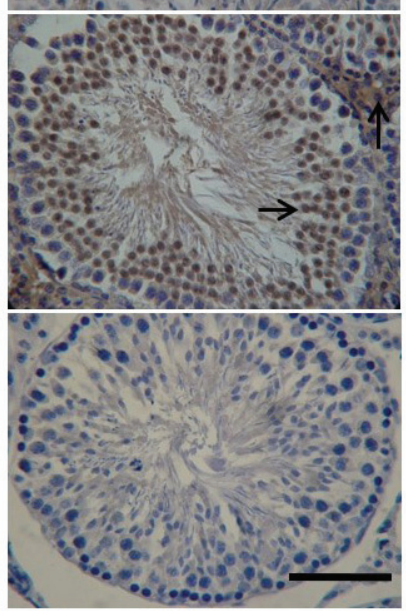

B
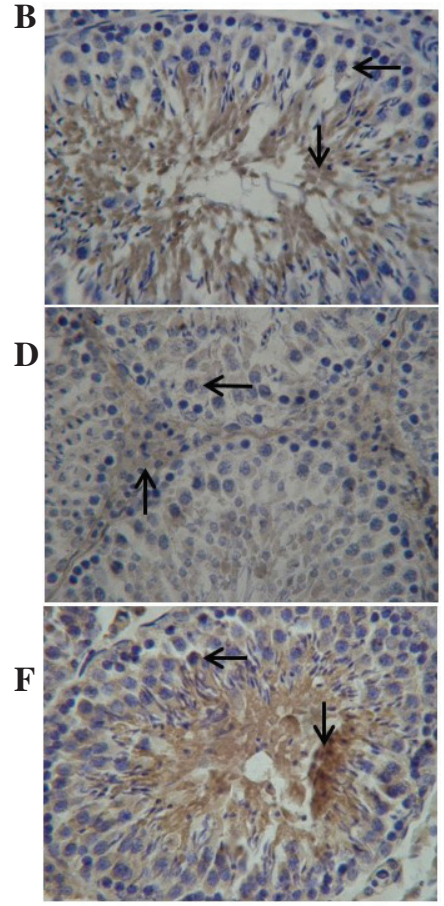

Figure 6. Immunohistochemical localization of IGF-1, PTEN, total Akt and FoxO1 in the testes of rats following water immersion and restraint stress. The immunohistochemical signals appear brown and the counterstained background appears blue in color. Immunohistochemical localization of (A and B) IGF-1, (C) PTEN, (D) total Akt and (E and F) FoxO1. (G) In control sections, bovine serum albumin was used instead of primary antibody. $\rightarrow$, spermatocyte; $\downarrow$, spermatid; $\uparrow$, interstitial tissue. Scale bar=50 $\mu \mathrm{m}$. IGF-1, insulin-like growth factor 1; PTEN, phosphatase and tensin homolog deleted on chromosome 10 ; FoxO1, forkhead box protein $\mathrm{O} 1$.

A

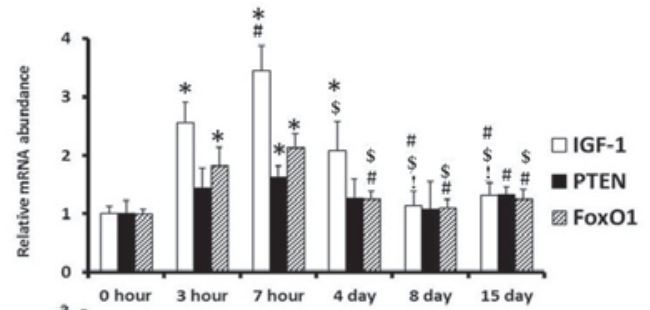

B

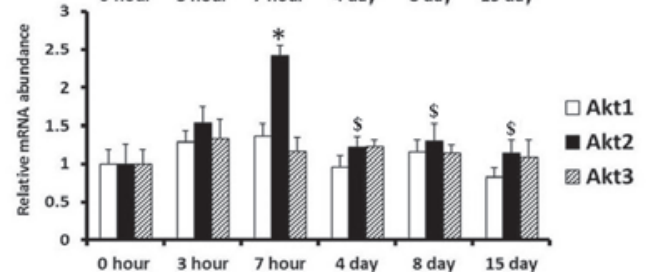

Figure 7. Relative expression levels of IGF-1, PTEN, Akt-1, Akt-2, Akt-3 and FoxOl in the testes of rats after WRS. (A) IGF-1, PTEN and FoxOI; (B) Akt1, $A k t 2$ and $A k t 3$ expression. $\mathrm{n}=6$ in each treatment group. ${ }^{*} \mathrm{P}<0.05$ vs. the $0 \mathrm{~h}$ group; ${ }^{*} \mathrm{P}<0.05$ vs. the $3 \mathrm{~h}$ group; ${ }^{\mathrm{S}} \mathrm{P}<0.05$ vs. the $7 \mathrm{~h}$ group; ${ }^{\mathrm{P}}<0.05$ vs. the 4 day group. WRS, water immersion and restraint stress; IGF-1, insulin-like growth factor 1; PTEN, phosphatase and tensin homolog deleted on chromosome 10; FoxO1, forkhead box protein $\mathrm{O}$.

cells via stimulation of PTEN activity. Moe-Behrens et al (37) reported estrogen exposure induced formation of germ cell testicular tumors via the Akt/PTEN signaling pathway. Similarly, Kimura et al indicated that PTEN gene knockout caused testiculoma (20). Furthermore, PTEN gene expression in fetal rat testes has been shown to be age-specific, thus suggesting that PTEN has an important role in fetal rat testis development (21). However, Huang et al reported that during mouse spermatogenesis, PTEN did not have a key role in spermatogenesis regulation (22). The present study demonstrated that PTEN and total Akt proteins were predominantly localized in the cytoplasm of Leydig cells and spermatogonia, and PTEN was also expressed in vascular endothelial cells. In addition, the results of a qPCR demonstrated that WRS increased gene expression levels of PTEN and Akt2 in rat testes, thus indicating that PTEN and Akt2 may be involved in the anti-stress mechanism of testes in rats subjected to WRS.

FoxO genes are the most important downstream target genes of the IGF-1/PTEN/Akt signaling pathway, which participate in several physiological processes, including cell differentiation, proliferation, apoptosis, migration and stress resistance (38). Our previous study demonstrated that FoxO3a and FoxO4 serve major roles in the digestive tract (27,39-41). A previous study regarding the male reproductive system demonstrated that FoxO1 served a key role in initiating spermatogenesis; however, FoxO3a and FoxO4 did not (39). 
Therefore, the expression and location of FoxO1 in WRS rat testes were determined. The results demonstrated that FoxO1 protein was widely expressed in the cytoplasm of spermatids, which were in the late stages of spermatogenesis and in testicular interstitial cells, thus implying that FoxO1 may be involved in spermatogenesis and cell apoptosis regulation in the late stages of spermatogenesis. Furthermore, WRS increased $\mathrm{FoxOl}$ gene expression levels in rat testes, indicating that the FoxOl gene may participate in the anti-stress mechanisms of rat testes.

In conclusion, WRS induced sperm injury in rat testes. These results suggested that the IGF-1/PTEN/Akt/FoxO signaling pathway may serve an anti-stress role in the testes of rats subjected to WRS. Future research will study the effect of IGF-1 on male reproduction and the involvement of the IGF-1/PTEN/Akt/FoxO signaling pathway.

\section{Acknowledgements}

The present study was supported by the National Nature Science Foundation of China (grant no. 81300287), the Senior Talents Scientific Research Foundation of Jiangsu University (grant no. 12JDG084) and the Nature Science Foundation of Jiangsu Province, China (grant nos. BK 2011499 and BK20140541).

\section{References}

1. Vazquez-Palacios G and Velazquez-Moctezuma J: Effect of electric foot shocks, immobilization and corticosterone administration on the sleep-wake pattern in the rat. Physiol Behav 71: 23-28, 2000.

2. Marin MT, Cruz FC and Planeta CS: Chronic restraint or variable stresses differently affect the behavior, corticosterone secretion and body weight in rats. Physiol Behav 90: 29-35, 2007.

3. Adachi M, Horiuchi G, Ikematsu N, Tanaka T, Terao J, Satouchi K and Tokumura A: Intragastrically administered lysophosphatidic acids protect against gastric ulcer in rats under water-immersion restraint stress. Dig Dis Sci 56: 2252-2261, 2011.

4. Nie SN, Qian XM, Wu XH, Yang SY, Tang WJ, Xu BH, Huang F, Lin X, Sun DY, Sun HC and Li ZS: Role of TFF in healing of stress-induced gastric lesions. World J Gastroenterol 9: 1772-1776, 2003.

5. Jiang P, Chang L, Pan CS, Qi YF and Tang CS: Protective role of metallothionein in stress-induced gastric ulcer in rats. World J Gastroenterol 11: 2739-2743, 2005.

6. Castrillon DH, Miao L, Kollipara R, Horner JW and DePinho RA: Suppression of ovarian follicle activation in mice by the transcription factor Foxo3a. Science 301: 215-218, 2003.

7. Reddy P, Liu L, Adhikari D, Jagarlamudi K, Rajareddy S, Shen Y, Du C, Tang W, Hämäläinen T, Peng SL, et al: Oocyte-specific deletion of Pten causes premature activation of the primordial follicle pool. Science 319: 611-613, 2008.

8. Carroll PV: Treatment with growth hormone and insulin-like growth factor-I in critical illness. Best Pract Res Clin Endocrinol Metab 15: 435-451, 2001.

9. Fresno Vara JA, Casado E, de Castro J, Cejas P, Belda-Iniesta C and González-Barón M: PI3K/Akt signalling pathway and cancer. Cancer Treat Rev 30: 193-204, 2004.

10. Leslie $\mathrm{N}$ and Downes C: PTEN function: How normal cells control it and tumour cells lose it. Biochem J 382: 1-11, 2004.

11. Sengupta A, Molkentin JD, Paik JH, DePinho RA and Yutzey KE: FoxO transcription factors promote cardiomyocyte survival upon induction of oxidative stress. J Biol Chem 286: 7468-7478, 2011.

12. Cross DA, Alessi DR, Cohen P, Andjelkovich M and Hemmings BA: Inhibition of glycogen synthase kinase- 3 by insulin mediated by protein kinase B. Nature 378: 785-789, 1995.

13. Burgering BM and Kops GJ: Cell cycle and death control: Long live Forkheads. Trends Biochem Sci 27: 352-360, 2002.
14. Watanabe S, Wang X, Hirose M, Kivilioto T, Osada T, Miwa H, Oide H, Kitamura T, Yoneta T, Seto K and Sato N: Insulin-like growth factor I plays a role in gastric wound healing: Evidence using a zinc derivative, polaprezinc, and an in vitro rabbit wound repair model. Aliment Pharmacol Ther 12: 1131-1138, 1998.

15. Colón E, Zaman F, Axelson M, Larsson O, Carlsson-Skwirut C, Svechnikov KV and Söder O: Insulin-like growth factor-I is an important antiapoptotic factor for rat leydig cells during postnatal development. Endocrinology 148: 128-139, 2007.

16. Khan SA, Ndjountche L, Pratchard L, Spicer L and Davis JS: Follicle-stimulating hormone amplifies insulin-like growth factor I-mediated activation of AKT/protein kinase B signaling in immature rat Sertoli cells. Endocrinology 143: 2259-2267, 2002.

17. Tai P, Shiraishi K and Ascoli M: Activation of the lutropin/choriogonadotropin receptor inhibits apoptosis of immature Leydig cells in primary culture. Endocrinology 150: 3766-3773, 2009.

18. Wu Y, Dowbenko D, Pisabarro MT, Dillard-Telm L, Koeppen H and Lasky LA: PTEN 2, a Golgi-associated testis-specific homologue of the PTEN tumor suppressor lipid phosphatase. J Biol Chem 276: 21745-21753, 2001.

19. Dupont J, Musnier A, Decourtye J, Boulo T, Lécureuil C, Guillou H, Valet S, Fouchécourt S, Pitetti JL, Nef S, et al: FSH-stimulated PTEN activity accounts for the lack of FSH mitogenic effect in prepubertal rat Sertoli cells. Mol Cell Endocrinol 315: 271-276, 2010.

20. Kimura T, Suzuki A, Fujita Y, Yomogida K, Lomeli H, Asada N, Ikeuchi M, Nagy A, Mak TW and Nakano T: Conditional loss of PTEN leads to testicular teratoma and enhances embryonic germ cell production. Development 130: 1691-1700, 2003.

21. Luukko K, Ylikorkala A, Tiainen M and Mäkelä TP: Expression of LKB1 and PTEN tumor suppressor genes during mouse embryonic development. Mech Dev 83: 187-190, 1999.

22. Huang Y, Mao X, Boyce T and Zhu GZ: Dispensable role of PTEN in mouse spermatogenesis. Cell Biol Int 35: 905-908, 2011.

23. Bagchi G, Zhang Y, Stanley KA and Waxman DJ: Complex modulation of androgen responsive gene expression by methoxyacetic acid. Reprod Biol Endocrinol 9: 42, 2011.

24. Goertz MJ, Wu Z, Gallardo TD, Hamra FK and Castrillon DH: Foxol is required in mouse spermatogonial stem cells for their maintenance and the initiation of spermatogenesis. J Clin Invest 121: 3456-3466, 2011.

25. John GB, Gallardo TD, Shirley LJ and Castrillon DH: Foxo3 is a PI3K-dependent molecular switch controlling the initiation of oocyte growth. Dev Biol 321: 197-204, 2008.

26. John GB, Shirley LJ, Gallardo TD and Castrillon DH: Specificity of the requirement for Foxo3 in primordial follicle activation. Reproduction 133: 855-863, 2007.

27. Huang P, Zhou Z, Wang H, Wei Q, Zhang L, Zhou X, Hutz RJ and Shi F: Effect of the IGF-1/PTEN/Akt/FoxO signaling pathway on the development and healing of water immersion and restraint stress-induced gastric ulcers in rats. Int J Mol Med 30: 650-658, 2012.

28. Wang H, Huang P, Lie T, Li J, Hutz RJ, Li K and Shi F: Reproductive toxicity of acrylamide-treated male rats. Reprod Toxicol 29: 225-230, 2010.

29. Kelly KJ, Sandoval RM, Dunn KW, Molitoris BA and Dagher PC: A novel method to determine specificity and sensitivity of the TUNEL reaction in the quantitation of apoptosis. Am J Physiol Cell Physiol 284: C1309-C1318, 2003.

30. Salimi A, Roudkenar MH, Sadeghi L, Mohseni A, Seydi E, Pirahmadi N and Pourahmad J: Ellagic acid, a polyphenolic compound, selectively induces ROS-mediated apoptosis in cancerous B-lymphocytes of CLL patients by directly targeting mitochondria. Redox Biol 6: 461-471, 2015.

31. Ding W, Wang W, Zhou B, Zhang W, Huang P, Shi F and Taya K: Formation of primordial follicles and immunolocalization of PTEN, PKB and FOXO3A proteins in the ovaries of fetal and neonatal pigs. J Reprod Dev 56: 162-168, 2010.

32. Livak KJ and Schmittgen TD: Analysis of relative gene expression data using real-time quantitative PCR and the 2(-Delta Delta C(T)) Method. Methods 25: 402-408, 2001.

33. Creagh EM, Conroy $\mathrm{H}$ and Martin SJ: Caspase-activation pathways in apoptosis and immunity. Immunol Rev 193: 10-21, 2003.

34. Rabinovsky ED: The multifunctional role of IGF-1 in peripheral nerve regeneration. Neurol Res 26: 204-210, 2004. 
35. Nguyen T, Chai J, Li A, Akahoshi T, Tanigawa $\mathrm{T}$ and Tarnawski AS: Novel roles of local insulin-like growth factor-1 activation in gastric ulcer healing: Promotes actin polymerization, cell proliferation, re-epithelialization, and induces cyclooxygenase-2 in a phosphatidylinositol 3-kinase-dependent manner. Am J Pathol 170: 1219-1228, 2007.

36. Colon E, Zaman F, Axelson M, Larsson O, Carlsson-Skwirut C, Svechnikov KV and Söder O: Insulin-like growth factor-I is an important antiapoptotic factor for rat leydig cells during postnatal development. Endocrinology 148: 128-139, 2007.

37. Moe-Behrens GH, Klinger FG, Eskild W, Grotmol T, Haugen TB and De Felici M: Akt/PTEN signaling mediates estrogen-dependent proliferation of primordial germ cells in vitro. Mol Endocrinol 17: 2630-2638, 2003.
38. Nakae J, Biggs WH III, Kitamura T, Cavenee WK, Wright CV, Arden KC and Accili D: Regulation of insulin action and pancreatic beta-cell function by mutated alleles of the gene encoding forkhead transcription factor Foxo1. Nat Genet 32: 245-253, 2002.

39. Huang P, Zhou ZQ, Huang RH, Zhou B, Wei QW and Shi FX: Age-dependent expression of forkhead box $\mathrm{O}$ proteins in the duodenum of rats. J Zhejiang Univ Sci B 12: 730-735, 2011.

40. Zhou ZQ, Wang T, Pan LM, Huang RH and Shi FX: FoxO4 is the main forkhead transcriptional factor localized in the gastrointestinal tracts of pigs. J Zhejiang Univ Sci B 8: 39-44, 2007.

41. Huang $P$, Zhou $Z$, Zheng $M$ and Shi F: Effect of the IGF-1/PTEN/Akt/FoxO signaling pathway in the duodenal mucosa of rats subjected to water immersion and restraint stress. Genet Mol Res 11: 4775-4788, 2012. 